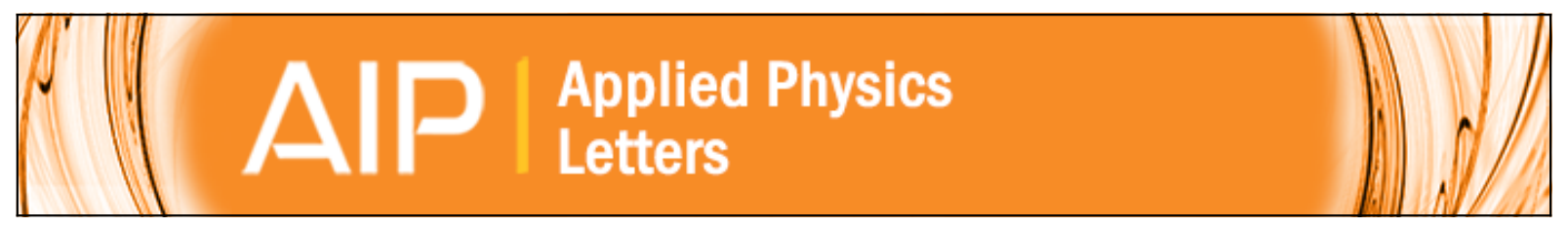

\title{
Recombination activity of interstitial iron and other transition metal point defects in $\mathbf{p}$ - and $\mathbf{n}$-type crystalline silicon
}

Daniel Macdonald and L. J. Geerligs

Citation: Applied Physics Letters 85, 4061 (2004); doi: 10.1063/1.1812833

View online: http://dx.doi.org/10.1063/1.1812833

View Table of Contents: http://scitation.aip.org/content/aip/journal/apl/85/18?ver=pdfcov

Published by the AIP Publishing

\section{Articles you may be interested in}

Charge states of the reactants in the hydrogen passivation of interstitial iron in P-type crystalline silicon J. Appl. Phys. 118, 085709 (2015); 10.1063/1.4929757

Impact of dopant compensation on the deactivation of boron-oxygen recombination centers in crystalline silicon Appl. Phys. Lett. 95, 232109 (2009); 10.1063/1.3272918

Recombination activity of iron-gallium and iron-indium pairs in silicon

J. Appl. Phys. 97, 113712 (2005); 10.1063/1.1929096

Spatial dependence of ultrafast carrier recombination centers of phosphorus-implanted and annealed silicon wafers

Appl. Phys. Lett. 81, 856 (2002); 10.1063/1.1497723

Surface photovoltage analysis of copper in $\mathrm{p}$-type silicon

Appl. Phys. Lett. 72, 2298 (1998); 10.1063/1.121341

\section{Horizon"OPO}

Tunable power and performance
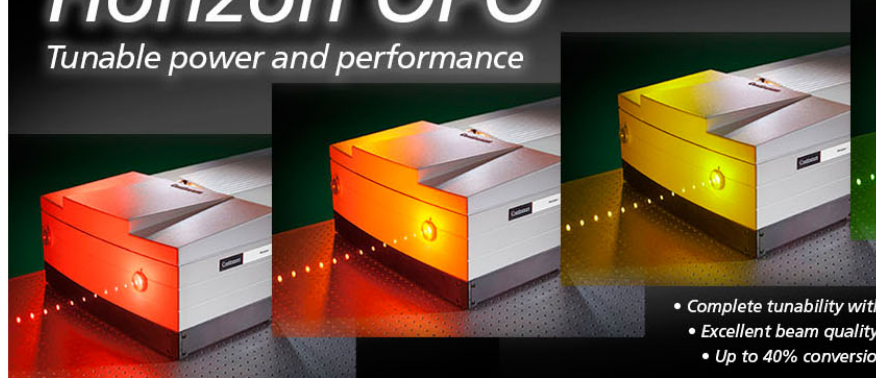

- Complete tunability with no degeneracy gap from 192-2750 nm - Excellent beam quality and low divergence in both axes - Up to $40 \%$ conversion efficiency

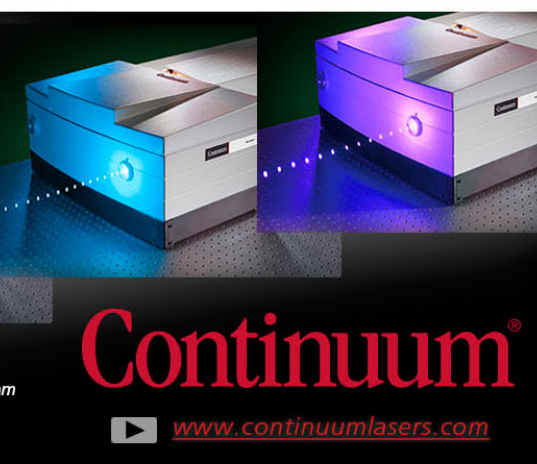




\title{
Recombination activity of interstitial iron and other transition metal point defects in $p$ - and $n$-type crystalline silicon
}

\author{
Daniel Macdonald ${ }^{\text {a) }}$ \\ Department of Engineering, FEIT, The Australian National University, Canberra, ACT 0200, Australia \\ L. J. Geerligs \\ ECN Solar Energy, PO Box 1, NL-1755 ZG Petten, The Netherlands
}

(Received 9 August 2004; accepted 1 September 2004)

\begin{abstract}
Interstitial iron in crystalline silicon has a much larger capture cross section for electrons than holes. According to the Shockley-Read-Hall model, the low-injection carrier lifetime in $p$-type silicon should therefore be much lower that in $n$-type silicon, while in high injection they should be equal. In this work we confirm this modeling using purposely iron-contaminated samples. A survey of other transition metal impurities in silicon reveals that those which tend to occupy interstitial sites at room temperature also have significantly larger capture cross sections for electrons. Since these are also the most probable metal point defects to occur during high temperature processing, using $n$-type wafers for devices such as solar cells may offer greater immunity to the effects of metal contaminants. @ 2004 American Institute of Physics. [DOI: 10.1063/1.1812833]
\end{abstract}

Metallic impurities are common in photovoltaic grade crystalline silicon. Neutron activation analysis ${ }^{1,2}$ studies of directionally solidified multicrystalline silicon have revealed, for example, significant quantities of $\mathrm{Fe}, \mathrm{Cr}, \mathrm{Cu}, \mathrm{Mo}$, and $\mathrm{Co}$. These contaminants are especially damaging when occurring at interstitial or substitutional lattice sites, since they may then be evenly distributed throughout the wafer. Precipitated metals may also be very active in terms of recombination, but at least in this case many impurity atoms are bound in one location.

Interstitial iron $\left(\mathrm{Fe}_{i}\right)$ is an especially common contaminant: it produces a deep level in the band gap and has a much larger capture cross section for electrons than for holes. ${ }^{3}$ According to the Shockley-Read-Hall (SRH) model, this implies that the low-injection carrier lifetime should be much lower in $p$-type silicon $(p-\mathrm{Si})$ than in $n$ type $(n-\mathrm{Si})$. The purpose of this work is to confirm this modeling experimentally. In addition, it is shown that other metal contaminants which tend not to precipitate during cooling, and are therefore more likely to produce point-like defects, also have larger cross sections for electrons. It is precisely these types of impurities which are the most dangerous for devices which are sensitive to the bulk carrier lifetime, such as solar cells.

The recombination strength of a given impurity is determined by three parameters: the energy level and the capture cross sections for both electrons and holes. Deep level transient spectroscopy (DLTS) has been applied to many metal impurities in silicon in recent decades, yielding much information about energy levels and majority carrier capture cross sections. In cases where minority capture was also studied, measurements were often performed well below room temperature (RT), and with strong electric fields (due to probing in the space-charge region). In only a few cases, such as for $\mathrm{Ti}$ and $\mathrm{Zn},{ }^{4,5}$ is the minority capture cross section also known from DLTS-like techniques under conditions applicable to the base region of solar cells (zero or small field and at RT).

${ }^{a}$ Author to whom correspondence should be addressed; electronic mail: daniel.macdonald@anu.edu.au
For some other impurities, the minority carrier cross sections under these conditions are known by carrier lifetime techniques. These values are collected in Table I for $\mathrm{Fe}^{3}{ }^{3} \mathrm{Cr}$, ${ }^{6,7}$ $\mathrm{Ti}^{4}{ }^{4} \mathrm{~V},{ }^{8,9} \mathrm{Mo},{ }^{10} \mathrm{Zn}^{5}$ and $\mathrm{Au} .{ }^{10}$ Values from Refs. 5 and 7 are based on capture coefficients $c_{n / p}$, converted to cross sections $\sigma_{n / p}$ using the thermal velocity ${ }^{11} v_{\text {th }}=1.1 \times 10^{7} \mathrm{~cm} / \mathrm{s}$.

For the case of $\mathrm{Fe}_{i}$, the ratio of electron to hole capture cross sections, $k=\sigma_{n} / \sigma_{p}$, is approximately 700 . Using these parameters, and the energy level, it is straightforward to model the injection dependence of the excess carrier lifetime with the SRH model. ${ }^{2-14}$ For deep levels such as $\mathrm{Fe}_{i}$, the low injection lifetime is limited by the capture of minority carriers, or electrons in $p$-Si. In high injection, however, the lifetime is controlled by both the electron and hole capture rates. If the hole capture cross section is much smaller, it will dominate the high injection lifetime, making it relatively large. Consequently, the lifetime will increase dramatically with injection level, as shown in Fig. 1. In $n$-Si, on the other hand, the lifetime is controlled by hole capture across the entire injection range, making it independent of injection. Note that the ratio of the low-injection lifetimes in $n$ - and $p$-Si is equal to $k$.

To verify the modeling, samples containing Fe were prepared by ion implantation with a dose of $10^{11} \mathrm{~cm}^{-2}$ and annealing at $900{ }^{\circ} \mathrm{C}$ for $1 \mathrm{~h}$. After etching, plasma-enhanced chemical vapor-deposited $\mathrm{SiN}$ films were deposited for surface passivation. The float-zone (FZ) $n$-Si samples were of resistivity $2 \Omega \mathrm{cm}\left(N_{D}=2.4 \times 10^{15} \mathrm{~cm}^{-3}\right)$ and $260 \mu \mathrm{m}$ thickness, giving a distributed $\mathrm{Fe}$ concentration of 3.8 $\times 10^{12} \mathrm{~cm}^{-3}$. The $p$-Si data are taken from Ref. 14, in which FZ wafers of $5 \Omega \mathrm{cm}\left(N_{A}=2.8 \times 10^{15} \mathrm{~cm}^{-3}\right)$ and $290 \mu \mathrm{m}$ thickness were used, giving a similar $\mathrm{Fe}$ concentration of $3.4 \times 10^{12} \mathrm{~cm}^{-3}$.

Effective carrier lifetimes $\tau$ were measured using the quasi-steady-state photoconductance technique. ${ }^{15}$ Control wafers without Fe implants were co-processed. By measuring the control and implanted wafers across the same injection range, the carrier lifetime due only to the introduced $\mathrm{Fe}_{i}\left(\tau_{\mathrm{Fe}}\right)$ can be extracted via $1 / \tau_{\mathrm{Fe}}=1 / \tau_{\text {implanted }}-1 / \tau_{\text {control }}$. This procedure subtracts out the influence of other recombi- 
TABLE I. Recombination parameters of point-like metal impurities in crystalline silicon at RT.

\begin{tabular}{llllclll}
\hline \hline Impurity & RT lattice site & \multicolumn{1}{c}{ Type } & Group & $\begin{array}{c}\text { Energy } \\
(\mathrm{eV})\end{array}$ & $\begin{array}{c}\sigma_{n} \\
\left(\mathrm{~cm}^{2}\right)\end{array}$ & $\begin{array}{c}\sigma_{p} \\
\left(\mathrm{~cm}^{2}\right)\end{array}$ & $k=\sigma_{n} / \sigma_{p}$ \\
\hline $\mathrm{Ti}$ & Interstitial & Donor & 4 & $E_{C^{-}}-0.27$ & $3.1 \times 10^{-14}$ & $1.4 \times 10^{15}$ & 22 \\
& Interstitial & D. donor & & $E_{V}+0.26$ & $1.3 \times 10^{-14}$ & $2.8 \times 10^{-17}$ & 460 \\
$\mathrm{~V}$ & Interstitial & D. donor & 5 & $E_{V}+0.36$ & $5 \times 10^{-14}$ & $3.0 \times 10^{-18}$ & 17000 \\
$\mathrm{Cr}$ & Interstitial & Donor & 6 & $E_{C}-0.22$ & $2.3 \times 10^{-13}$ & $1.1 \times 10^{-13}$ & 2 \\
$\mathrm{Mo}$ & Interstitial & Donor & 6 & $E_{V}+0.28$ & $1.6 \times 10^{-14}$ & $6.0 \times 10^{-16}$ & 30 \\
$\mathrm{Fe}$ & Interstitial & Donor & 8 & $E_{V}+0.38$ & $5 \times 10^{-14}$ & $7 \times 10^{-17}$ & 700 \\
$\mathrm{Au}$ & Substitutional & Acceptor & 11 & $E_{C}-0.55$ & $1.4 \times 10^{-16}$ & $7.6 \times 10^{-15}$ & 0.02 \\
$\mathrm{Zn}$ & Substitutional & Acceptor & 12 & $E_{V}+0.33$ & $1.5 \times 10^{-15}$ & $4.4 \times 10^{-15}$ & 0.34 \\
& Substitutional & D. acceptor & & $E_{C}-0.47$ & $1.3 \times 10^{-19}$ & $6.6 \times 10^{-15}$ & 0.00002 \\
\hline \hline
\end{tabular}

nation channels such as Auger and surface recombination. Figure 2 shows the result for the Fe-doped $n$-Si wafer. Figure 1 shows the results after the subtraction procedure for both $n$ - and $p$-Si. The data for the boron-doped $p$-Si sample were taken after illumination to break the FeB pairs, ${ }_{16}^{16}$ which display an entirely different injection dependence. ${ }^{14}$

The agreement between the model and the measured data is very good, especially considering the $\mathrm{Fe}_{i}$ concentration is determined by the dose, and is not a free parameter. Slight reforming of $\mathrm{FeB}$ pairs (estimated to be approximately $10 \%$ ) before measurement is probably the reason for the disagreement at higher injection levels for the $p$-type case. From this data we can extract a lower bound for the capture cross section ratio for $\mathrm{Fe}_{i}$ of $k>75$. The lifetime could not be measured at lower injection levels in the $p$-Si sample due to minority carrier trapping artifacts.

Many of the other transition metal impurities in Table I also have $k$ values far from unity. Figure 3 plots $k$ as a function of the energy gap to the nearest band edge. The plot shows a striking trend: the donor levels all have $k>1$, while the acceptor levels have $k<1$. As recombination centers, donor levels alternate between a positively charged state which captures electrons, and a neutral state which captures holes. Since the charged state is more attractive to electrons than the neutral state is to holes, it seems reasonable to have $k>1$. The opposite occurs for the acceptor levels. Note also that double donors and acceptors, which are doubly charged, have even more extreme $k$ values.

Graff $^{10}$ and Lemke ${ }^{17}$ have noted that, in general, metals from Group 8 or less on the periodic table are more likely to produce donor states, while those from Group 9 or higher generally produce acceptor states, as reflected in Table I. There are numerous exceptions to this trend, for example, Ti and V (Groups 4 and 5) exhibit both donor and acceptor states. However, their acceptor states are quite shallow, ${ }^{10}$ and

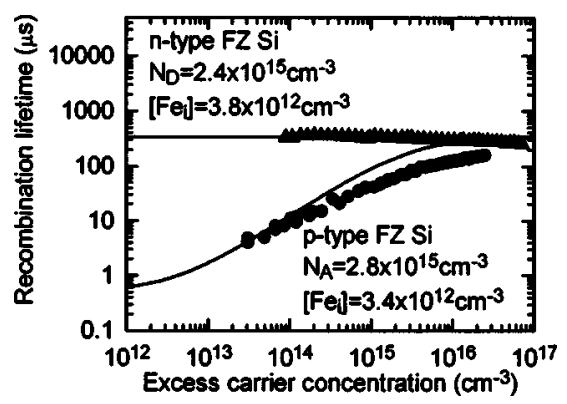

FIG. 1. Modeled and measured lifetimes for $\mathrm{Fe}_{i}$ in $n$ - and $p$-type silicon. are unlikely to dominate the recombination activity of these metals. Hence it is generally true that the most recombination-active levels of transition metals from Group 8 and below are donor states.

Graff $^{10}$ and Lemke ${ }^{17}$ have also observed that transition metals from Group 8 or below tend to form moderate concentrations of interstitial centers at RT after high temperature processing, while those from above Group 8 generally form precipitates and much lower concentrations of substitutional centers. This occurs because the diffusivities of the latter are significantly higher. Since their diffusion length is still high at temperatures where the solubility becomes extremely small, almost complete precipitation of the interstitial atoms occurs. As a result, only the very small fraction which occupied substitutional sites prior to quenching remains in solution. Such behavior is well known for $\mathrm{Co}, \mathrm{Ni}$, and $\mathrm{Cu}$ (Groups 9, 10 and 11), for example. ${ }^{18}$

Thus there is an apparent coincidence in Table I: metals from Group 8 and below are both donor-like and interstitial at RT, whereas those from above Group 8 are acceptor-like and substitutional at RT. As a result of being donor-like, the interstitial metals have large values of $k$, while the RT substitutional metals are acceptor-like with $k \ll 1$. This observation has profound implications for their recombination activity: the interstitial metals are, generally, more detrimental in $p$-Si, while the substitutional metals have a greater impact in $n$-Si. Itsumi ${ }^{19}$ came to similar conclusions for the specific cases of $\mathrm{Fe}, \mathrm{Ni}$ and $\mathrm{Cu}$. Since the interstitial impurities are likely to occur in much higher concentrations than the substitutional ones (from the precipitation argument above), they are the most important point-like metal contaminants technologically. Notwithstanding the impact of precipitates, whose relative recombination activity in $n$ - and $p$-Si is not well

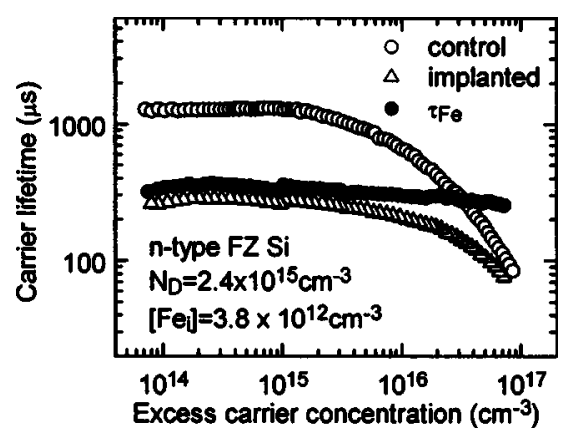

FIG. 2. Measured control and implanted lifetimes and calculated lifetime due to $\mathrm{Fe}_{i}$ only $\left(\tau_{\mathrm{Fe}}\right)$ for the $n$-Si sample. 


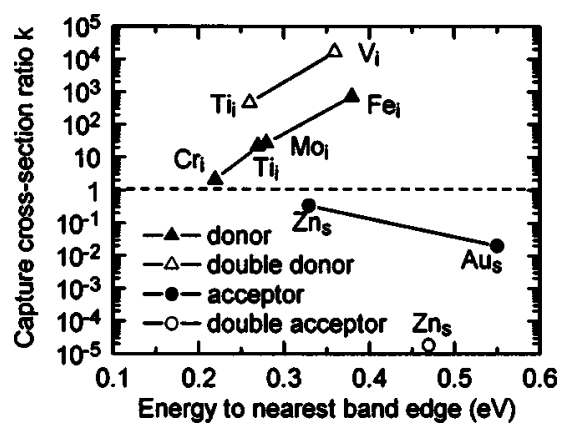

FIG. 3. Capture cross section ratio as a function of energy level depth for impurities in Table I.

established, this supports the use of $n$-Si for devices such as solar cells. It may also explain the recently observed very high lifetimes in $n$-type multicrystalline silicon. ${ }^{20}$

A further observation from Fig. 3 is that deeper energy levels have greater values of $k$. This is less easily explained, but is related to the details of the capture process, since cascade, multi-phonon or impurity-mediated Auger mechanisms lead to different models for how capture cross sections depend on the defect depth. ${ }^{21}$ It can also be observed from Table I that, for the case of donor states, the major part of this effect is due to the variation in $\sigma_{p}$, while $\sigma_{n}$ is relatively constant. This may be related to the fact that, for donor levels, the degree of Coulombic attraction for electrons is not influenced strongly by the level depth, while the transition probability for a neutral (hole) capture process will be.

Although "deep" levels with $k>1$ will display the same general injection-dependent behavior as $\mathrm{Fe}_{i}$ in Fig. 1, shallower levels can be more complex due to interactions with the Fermi level. They may have an injection dependence that either increases or decreases, depending on the resistivity. ${ }^{14}$ Since many of the other levels in Table I are not especially deep, it is important to apply the SRH model for each to determine if they result in a "preference" for $n$-Si over $p$-Si. With $N_{A / D}=1 \times 10^{16} \mathrm{~cm}^{-3}$, it is indeed true that $\mathrm{V}$, Mo and both levels of Ti produce much greater recombination in $p$ -Si. The opposite is true for the acceptor levels of $\mathrm{Au}$ and $\mathrm{Zn}$.

Naturally, the performance of a solar cell depends on many parameters other than the low-injection lifetime in the base. First, it is often the diffusion length $L_{D}$, rather than carrier lifetime $\tau$, that determines the cell current. Since the minority carrier diffusivity $D$ in $n$-Si is typically a factor of 3 lower than in $p$-Si, the lifetime must increase by a factor of 3 to achieve the same diffusion length $\left(L_{D}=[D \tau]^{1 / 2}\right)$. Note that, except for $\mathrm{Cr}$, all of the interstitial impurities shown in Table I have $k>3$. Another consideration is that solar cells generally operate at moderate injection where the difference between $n$ - and $p$-type lifetimes is not as large as in true low injection. In addition, recombination in other parts of a device may mask the impact of base recombination. It is also known that interstitial impurities can be removed by the get- tering action of highly doped regions, and some metals, such as $\mathrm{Fe}_{i}$, are effectively hydrogenated during cell processing. ${ }^{22}$ Nevertheless, industrial phosphorus diffusions are often too rapid to allow complete gettering, and similarly hydrogenation may be incomplete or ineffective for some metals. It is therefore conceivable that interstitial metals will, in some cases, impact on cell performance.

In conclusion, transition metal contaminants from Group 8 and below on the periodic table are the most likely to produce relatively large concentrations of point defect recombination centers in crystalline silicon. These are interstitial in nature, and correspond to those metals which tend to produce donor states, and therefore have much greater recombination strength in $p$-Si than $n$-Si. If the presence of these impurities dominates cell performance, then $n$-Si may prove to be a better choice of solar cell substrate.

This work has been supported by the Australian Research Council and The Netherlands Agency for Energy and the Environment.

${ }^{1}$ A. A. Istratov, T. Buonassisi, R. J. McDonald, A. R. Smith, R. Schindler, J. A. Rand, J. P. Kalejs, and E. R. Webber, J. Appl. Phys. 94, 6552 (2003).

${ }^{2}$ D. Macdonald, A. Cuevas, A. Kinomura, and Y. Nakano, in Proceedings of the 29th Photovoltaic Specialists Conference New Orleans, LA (IEEE, New York, 2002), p. 285.

${ }^{3}$ A. A. Istratov, H. Hieslmair, and E. R. Weber, Appl. Phys. A: Mater. Sci. Process. 69, 13 (1999).

${ }^{4}$ A. C. Wang, L. S. Lu, and C. T. Sah, Phys. Rev. B 30, 5896 (1984).

${ }^{5}$ C. T. Sah, P. C. H. Chan, C. K. Wang, R. L. Y. Sah, K. A. Yamakawa, and R. Lutwack, IEEE Trans. Electron Devices 28, 304 (1981).

${ }^{6}$ H. Conzelmann, K. Graff, and E. R. Weber, Appl. Phys. A: Solids Surf. 30, 169 (1983).

${ }^{7}$ K. Mishra, Appl. Phys. Lett. 68, 3281 (1996).

${ }^{8}$ L. Tilly, H. G. Grimmeiss, H. Pettersson, K. Schmalz, K. Tittelbach, and H. Kerkow, Phys. Rev. B 44, 12809 (1991).

${ }^{9}$ J. P. Kalejs, B. R. Bathey, J. T. Borenstein, and R. W. Stormont, in Conference Record of the 23rd IEEE Photovoltaic Specialists Conference, Louisville, KY (IEEE, New York, 1993), p. 184.

${ }^{10} \mathrm{~K}$. Graff, Metal Impurities in Silicon Device Fabrication, Springer Series in Materials Science, 2nd ed., (Springer, Verlag, Berlin, 2000).

${ }^{11}$ W. M. Bullis and H. R. Huff, J. Electrochem. Soc. 143, 1399 (1996).

${ }^{12}$ W. Shockley and W. T. Read, Phys. Rev. 87, 835 (1952).

${ }^{13}$ R. N. Hall, Phys. Rev. 87, 387 (1952).

${ }^{14}$ D. Macdonald, A. Cuevas, and J. Wong-Leung, J. Appl. Phys. 89, 7932 (2001).

${ }^{15}$ R. A. Sinton and A. Cuevas, Appl. Phys. Lett. 69, 2510 (1996).

${ }^{16}$ G. Zoth and W. Bergholz, J. Appl. Phys. 67, 6764 (1990).

${ }^{17}$ H. Lemke, in Semiconductor Silicon/1994, edited by H. R. Huff, W. Bergholz, and K. Sumino (Electrochemical Society, Pennington, NJ, 1994) p. 695.

${ }^{18}$ A. A. Istratov and E. R. Weber, Appl. Phys. A: Mater. Sci. Process. 66, 123 (1998).

${ }^{19}$ M. Itsumi, Appl. Phys. Lett. 63, 1095 (1993).

${ }^{20}$ A. Cuevas, M. J. Kerr, C. Samundsett, F. Ferrazza, and G. Coletti, Appl. Phys. Lett. 81, 4592 (2002).

${ }^{21}$ A. Hangleiter, Phys. Rev. B 37, 2594 (1988).

${ }^{22}$ A. Azzizi, L. J. Geerligs, and D. Macdonald, to be published in Proceedings of the 19th European Photovoltaic Solar Energy Conference, Paris, 2004. 